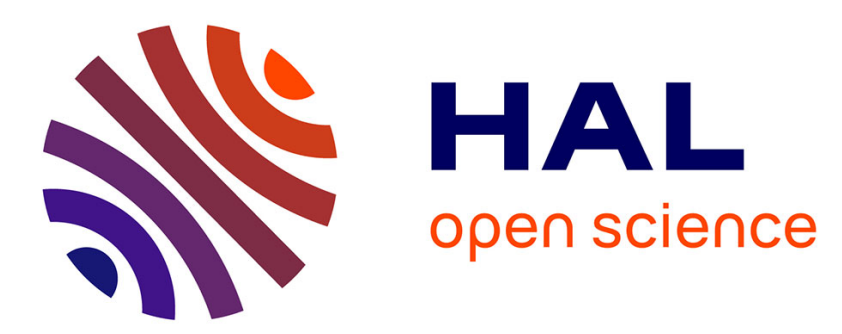

\title{
Establishing enterprise Business Context (eBC) for service policy decision in mobile broadband networks
}

\author{
Rebecca Copeland, Noel Crespi
}

\section{To cite this version:}

Rebecca Copeland, Noel Crespi. Establishing enterprise Business Context (eBC) for service policy decision in mobile broadband networks. ContextQoS ' 12: 2nd International Workshop on Contextaware QoS Provisioning and Management for Emerging Networks, Applications and Services, Jul 2012, Munich, Germany. pp.1-5, 10.1109/ICCCN.2012.6289204 . hal-00731273

\section{HAL Id: hal-00731273 \\ https://hal.science/hal-00731273}

Submitted on 12 Sep 2012

HAL is a multi-disciplinary open access archive for the deposit and dissemination of scientific research documents, whether they are published or not. The documents may come from teaching and research institutions in France or abroad, or from public or private research centers.
L'archive ouverte pluridisciplinaire HAL, est destinée au dépôt et à la diffusion de documents scientifiques de niveau recherche, publiés ou non, émanant des établissements d'enseignement et de recherche français ou étrangers, des laboratoires publics ou privés. 


\section{Establishing enterprise Business Context (eBC) for service policy decision in mobile broadband networks}

\author{
Rebecca Copeland, Core Viewpoint Ltd, Kenilworth, \\ United Kingdom (Author)
}

\begin{abstract}
$\square$ empowering the enterprise to control their own session policy for mobile broadband is not only necessary for consumerization, but for the enterprise to control costs and protect corporate network resources. We propose a practical method of establishing dynamically enterprise-Business-Context (eBC) status to determine whether or not the enterprise should fund employees $\square$ service requests and what QoS and charging levels should be assigned. To do that, the enterprise can use context sources that are not available externally and apply corporate business objectives. This paper describes the eBC Function, platform, processing logic, data sources and call flows, and details the computation method.
\end{abstract}

Keywords-Policy; Context; Charging Rules; Consumerisation; PCRF; Policy Decision; QoS Profiles; Role based access; SLA;

\section{INTRODUCTION}

\section{A. The concept of Enterprise Business Context (eBC)}

Consumerization is a growing trend in the enterprise sector, where companies allow employees to use their own devices for business. This extends to publically available web-based applications that are utilized for business, instead of growing the corporate application farm. While this saves considerable costs, the enterprise still needs to recompense employees for legitimate business use. Enterprises also need to protect their network resources that may be swamped by employees' smart devices. To address this issue, a new enterprise-BusinessContext (eBC) Function is proposed. It determines whether service requests are 'personal', for users to bear the costs via their personal subscriptions, or the requests are granted $\mathrm{eBC}$ status and are funded by the enterprise, in which case the eBC Function also determines QoS (Quality of Service) and charging levels for the service delivery.

Defining QoS levels for Internet services according to user personal and business identities has been studied in the NETQOS project [4], though the aim there was to unify policies, not to distinguish between business and personal use. Using context for brokering applications or modules is often proposed, as in [8] - for the web world. In the Mobile world, using 3GPP Policy for context-routing in Messaging (see [7]) is proposed. The eBC Function also interfaces to standard 3GPP PCC (Policy \& Charging Control) ([2]), but uses enterprise special, confidential sources to determine context.

\section{B. Benefits from Establishing Dynamic Business Context}

The eBC function empowers the enterprise to take control of its resources and address aspects of budgeting. The prime advantage of the eBC Function is enabling the enterprise to drive business objectives, prioritize resources and control

\author{
Noel Crespi, Telecom Sud-Paris, Evry, Paris, France \\ (co-Author)
}

levels of funding, much more than can be achieved by SLAs (Service Level Agreements). Using the eBC Function, the enterprise can encourage desirable behavior and preferred business practices, e.g. home working and team collaboration. It can accommodate job levels and seniority by increasing their $\mathrm{eBC}$ priorities. The enterprise will benefit from funding expenses directly without having to process expense claims. As these are auditable business expenditure, they should be acceptable to the tax man, benefitting both enterprise and employees. In fact all stakeholders benefit: Employees get better services, more funding and no hassle with expense claims. Department heads get better budget control and higher employee efficiency. ICT managers can optimize SLAs and resource allocation. Administrators can simplify expenses. Different operators get a 'bite' at the enterprise 'cherry' that otherwise would go to a single contract. Carriers can offload the complex context-based policy decision to the enterprise, reducing their overheads. ASPs (Apps Service Providers), including Cloud providers, get funded by the enterprise, and need no additional user screening.

Alternative ways do not achieve these aims. Enterprises could ignore consumerization, but this leads to unrestrained usage of enterprise resources, resulting in costly network upgrades. Enterprises may choose to reimburse employees' costs against receipts, but this increases bureaucracy and annoys the staff. Paying employees a flat rate is not the answer either - it will be taxed as taxable benefits. If left to the carrier, context would not be refined, enterprise strategies would not be conveyed and changes would not be updated promptly.

\section{Related work}

This paper is part of Enterprise Policy and Business Context study for mobile networks. Enterprise-controlled policy decision is described in another paper. The Policy needs an eBC Function, which is described here. The eBC computational Model and the Attributes aggregation are subject to other papers.

Part II in this paper describes the eBC platform, connectivity and data sources. Part III defines eBC logic and procedures. Part IV introduces the $\mathrm{eBC}$ Model and components, and details the eBC evaluation process. Conclusions are given in Part V.

\section{THE EBC FUNCTION IN THE NETWORK}

\section{A. Dealing with the Value Chain}

The eBC platform connects to external entities to receive context data and triggers from access nodes and filtering results from monitoring nodes. Figure 1 shows the eBC interaction 
with other entities. Context information may be available from carriers or ASPs. With all requests analyzed by the enterprise, SLAs can be tracked internally, but carriers' reports of SLA status should be obtained for comparison. Context information between entities could be shared over XACML (see [10]). Although XACML has no notion of a session, in this case, all that is needed is a one-off transfer of information.

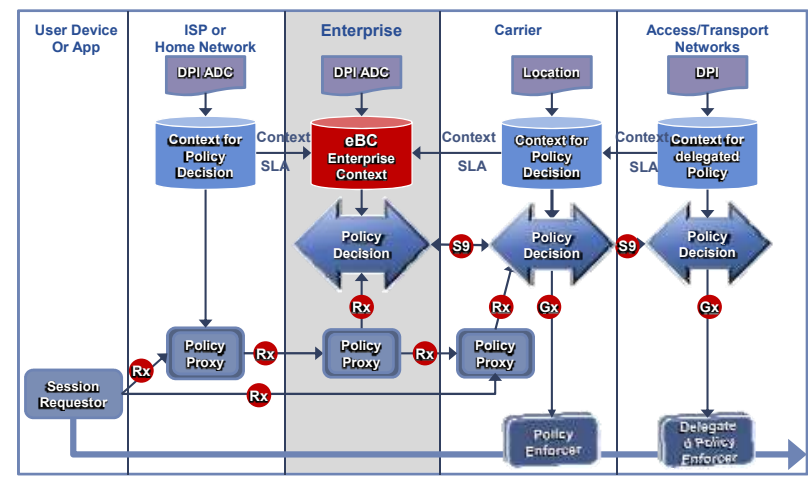

Figure 1: Heterogeneous Architecture for Context and Policies Decision

The enterprise needs a Policy Proxy function that formats the information into standard interfaces - Rx and S9 (see [2]). Service requests can reach the enterprise proxy in several ways: directly from the terminal, re-routed via the ASP or intercepted by DPI (Deep Packet Inspection) and ADC (Application Detection \& Control). As shown the ADC could be within the enterprise or at the carrier's network, or both. These elements are crucial to triggering, monitoring and reporting on the session, which are important sources of context.

\section{B. The Platform}

In [3], a carrier solution for an XACML based service orchestration is hinged on (1) Policy Proxy (2) Context Handler (3) Policy Server. As shown in Figure 2, similar architecture is adopted here. The eBC Function (as Context Handler) tracks SLAs per carrier and handles static context information (e.g. quotas). It interfaces to the Policy Proxy that receives and forwards service requests. It collaborates with the Policy Function for the preparation of the PCC rules.

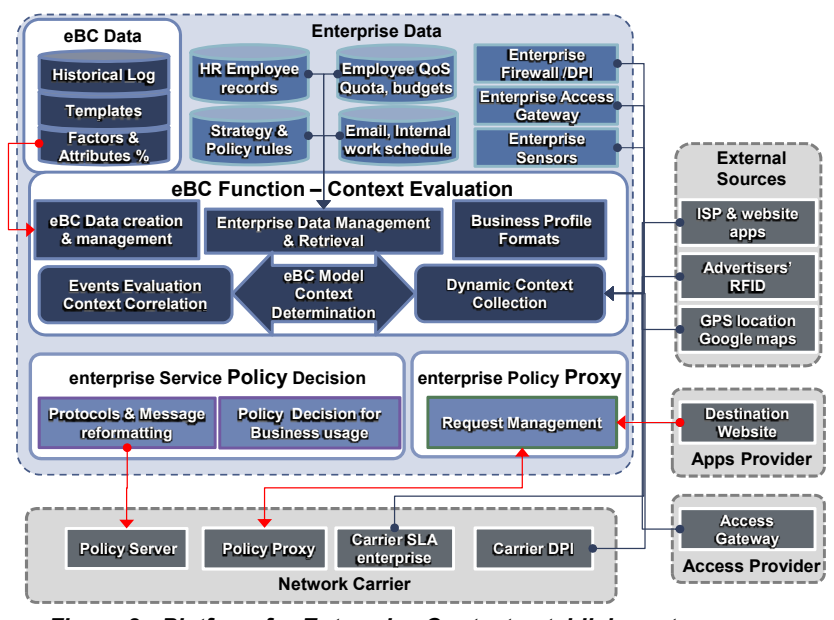

Figure 2: Platform for Enterprise Context establishment

The main engine is the eBC Model that calculates context status from collected data and priorities. The platform also contains data management module and data creation to configure the model. The eBC data creation must be simple and intuitive, for enterprise staff to create scenarios, templates and priority ratios. Any changes must be tested instantaneously via a set of representative scenarios. The context data collection module links to internal and external data sources and is also responsible for collection of data from sensors, geographic location positioning (GPS) and the like.

\section{Enterprise sources of context data}

The enterprise has numerous context data sources, both static and dynamic. These sources are not available outside the enterprise. For example, the enterprise can examine employees' diaries to ascertain currently booked engagements and business tasks, or confirm location via appointment venues. Context data is also retrieved from departmental budgets, list of enterprise sites etc. Important sources of information are the corporate work scheduling, shift roster, email system and the internal LAN login.

As working time and leisure time get mingled, retaining historical log of users' behavior can provide another dimension of work patterns and business practices. Previous eBC decisions can be 'learnt' and re-used. Travelling patterns can provide predictive context. This is rich ground for 'Policy Continuum' and the DEN-ng model [6], combining both dynamic data and accumulated knowledge in the eBC process.

Access to such confidential data that is not available to carriers is what makes the eBC Function so powerful. Privacy constraints that affect the highly regulated Telecom are not an issue for the enterprise. Privacy is a great concern in the web world too. For example, [8] proposes to combat privacy 'leakage' by forwarding access policy within service composition algorithms. In the enterprise case, it is entitled to use employee data stored within its systems, as long as data is not divulged to external parties and only the resulting decision is conveyed to the carriers.

\section{THE EBC PROCEDURE AND CALL FLOW}

\section{A. The eBC Evaluation procedure logic}

In [6], linking business concerns and network operations is explored, proving that semantic logic and ontology can bridge the gap between them. This is also the main aim here. The eBC logic analyzes raw context data and applies a set of filters, conditions and weighting ratios that are instrumental in interpreting the enterprise's objectives.

The logic flow, as shown in Figure 3, is designed to minimize unnecessary real-time data collection and save computation time. To this end, the request tuple details with static pre-stored context data are assessed first. This provides for cases where eBC status cannot be granted anyway (e.g. SLA not permitting) or where it is always granted regardless of context (e.g. CEO's request). In all other cases, the dynamic context collection module is activated and context data is analyzed. The eBC Model computes the score for each template that is allowed for this Role, to reduce computation time. If none of the templates has a score above the pre-set threshold, eBC status is not granted and the session is 
forwarded to the carrier as it is. If eBC status is granted, employee's policies and grades together with the chosen template are used to construct the session policy.

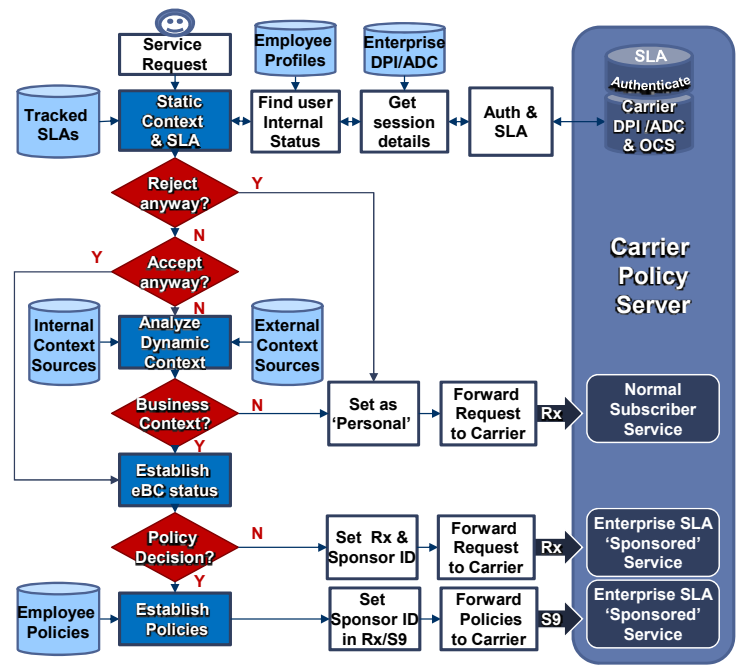

Figure 3: Logic flow for establishing eBC-based Policy

\section{B. Options for eBC interface to a Policy Server}

To enforce the $\mathrm{eBC}$ results, they must be conveyed to the network operator. This can be achieved by using the enterprise policy function to produce the required PCC rule and sending it to the carrier over the network-to-network interface (S9), which is specified by 3GPP in [2]. Since the enterprise is not a licensed virtual network, this requires the carrier to accept S9 information but process it against the enterprise SLA. If the carrier will not accept $\mathrm{S} 9$, the eBC results are used to reformulate the $\mathrm{Rx}$ request, indicating the decided QoS and charging levels. In both cases, the enterprise can act under a Sponsoring [2] SLA with the carrier, which allows the enterprise greater leeway in determining session policies.

\section{Call Flows for eBC evaluation}

Despite adhering to standard interfaces, the evaluation of eBC status requires some new call flows. Exchanging context information also needs to be formalized. Figure 4 provides a high level call flow for service request handling between the value chain parties (new interactions are in red). Session requests can arrive from alternative origins. SLA details should be obtained from the enterprise internal SLA tracking. These details and the request tuple are evaluated first. The Policy Proxy sends the received request to the Policy function, which calls upon the eBC Function to determine how to treat it.

Five possible options are illustrated. Option 1 is for requests that are always forwarded as personal use, and Option 2 is for those that are always accepted, regardless of context. Option 3 is exercised when there is no eBC Template value above the threshold and the original service request is forwarded unchanged to the carrier. When eBC is granted, Option 4 modifies the service request according to the template, while Option 5 sets up the final policy rule on the S9 interface. Option 4 and 5 depend on carriers' acceptance of S9 from the enterprise.

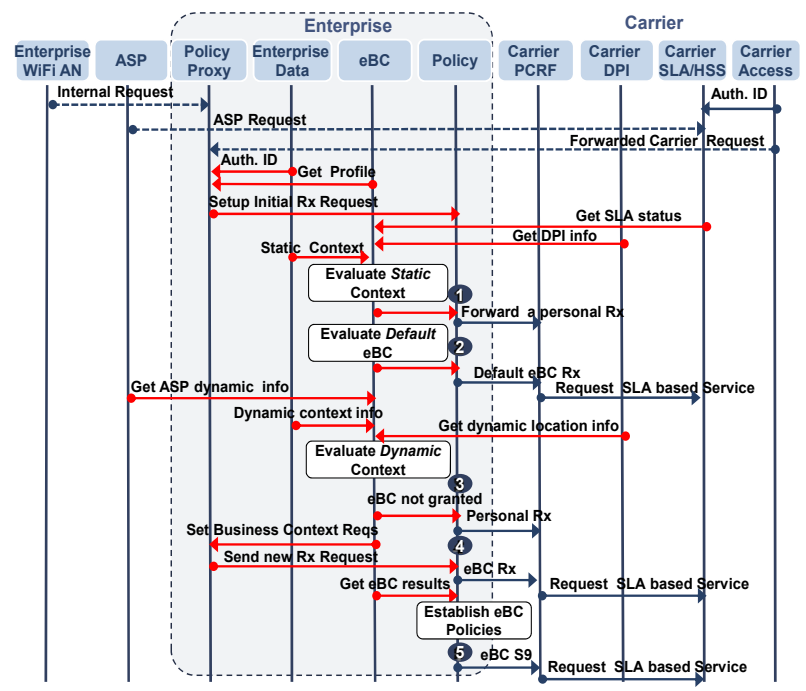

Figure 4: Call Flow for Service Request Processing

\section{The EBC Evaluation}

\section{A. The evaluated elements}

In [1], sharing personal context information is controlled via a policy hierarchy: enterprise $>$ team $>$ activity $>$ role $>$ user, where enterprise rules can be overturned by user rules. Obviously, for the purpose of enterprise controlling its resources and spending, the order is reversed. We propose a hierarchy where each element influences the next to be processed: Role $>$ Tasks $>$ Factors $>$ Attributes. Figure 5 shows the structure of the eBC definition. The Role defines what Tasks, Task defines Factors and Factors define Attributes. The values of these Attributes make the Factor, Factors are assed up in the Task Templates, and this determines the prevailing Task and its Policy Rules.

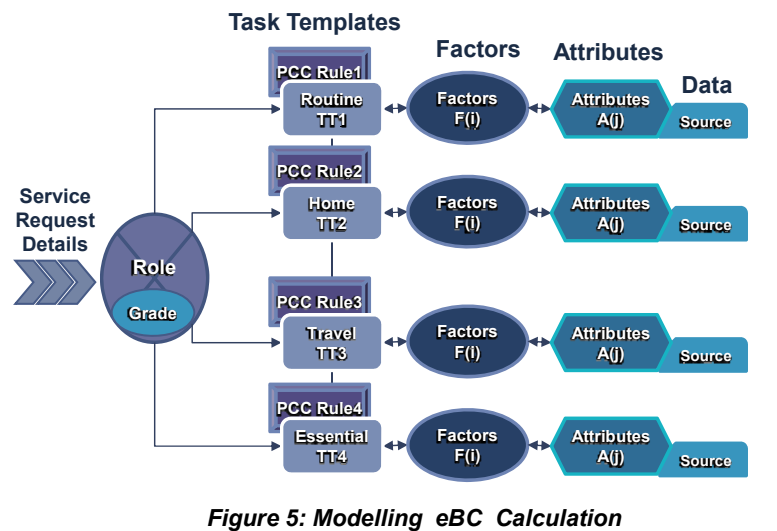

Both roles and tasks play a major part, but they affect the evaluation differently. The Role, which is a known element (for an authenticated user), shapes the evaluation by determining which Task Templates are processed, then modifies the final outcome via grade based privileges. The engaged task is not known and has to be 'discovered' via the context analysis of the Task Templates. The discovered prevailing Task Template defines a set of QoS and funding rules appropriate to this task. 


\section{B. Roles and Grades}

RBAC (Role Based Access Control) for access permission is featured in many papers, one of which is [13], and has been widely implemented, by the evidence of [12]. Examples of enterprise roles can be executive, administrator, shop floor worker or travelling sales, depending on the type of business. They have different sets of privileges, quota and budgets, according to the job content. Roles have assigned relevant Task Templates, e.g. the Role of shop-floor worker is not permitted to have the Travel-Abroad template. Grades within each role allow for fine-tuning of privileges, e.g. senior administrator compared with a junior clerk.

\section{Task Templates}

As Roles are not sufficient to define session features, we propose to use tasks as well. The concept of Task-Based Access Control (TBAC), as introduced in [14], was explored in [5] for Policy Based Access Control (PBAC) in workflow management. For eBC, the task needs to be ascertained first. To do that, regularly occurring tasks are formulated into templates. A Task Template, such as Travel or Home Working, is immediately recognizable and intuitively linked with common business scenarios. The Task Template contains Factors (location, working hours and destination) that are weighted according to their importance for the Task. For example location is a prime factor in the Travel Task. The weighting of Factors means that configured templates for the tasks return different values for the same scenario with the same Attribute evidence. This enables identifying the prevailing Task with the highest score.

Employee's request (n) has Role (Rn), Grade (Gn) and permitted Task Templates (TTn), which has some or all members of TT.

$$
\begin{aligned}
& \mathrm{TT}:=\{\mathrm{TT} 1, \mathrm{TT} 2 \ldots \mathrm{TTk}\} \quad \mathrm{TTn}:=\text { prevailing task for (n) } \\
& \mathrm{TTn} \in \mathrm{Rn} \quad \mathrm{TTn} \subseteq \mathrm{TT}
\end{aligned}
$$

\section{Factors}

While most published context models consider roles, tasks and attributes, we have introduced the concept of Factors. Factors are influencing aspects of Tasks that bridge between disparate sources of context data and the enterprise policies. The Factors weighting characterizes Task Templates. A Factor is defined by one or more Attributes, with only one providing the decisive result. A Task Template (TT) has Factors (Fi) which are built from Attributes $(\mathrm{Aj})$,

$$
\begin{aligned}
& \text { where } 0 \leq \mathrm{Fi} \geq 10 \quad 0 \leq \mathrm{Aj} \geq 10 \\
& \mathrm{~F}=\{\mathrm{F} 1, \mathrm{~F} 2 \ldots \mathrm{Fi}\} \wedge \mathrm{A}=\{\mathrm{A} 1, \mathrm{~A} 2 \ldots \mathrm{Aj}\} \rightarrow \mathrm{Fi}=\int(\mathrm{Aj})
\end{aligned}
$$

The Factor logic selects the prevailing Attribute. The Factor is made of exclusive (either-or) list of Attributes, or a list of overlapping alternatives. In the latter case, the highest probability (PAj) is accepted. For example, in the Time Factor, Attribute which is 'very near' working hours is considered stronger than flexi-time for the Routine Task.

Figure 6 has been generated by the eBC Model prototype. It shows Factor values in different scenarios, to demonstrate the effects on the Task Template. In the scenario 'Essential $24 \times 7$ ', which denotes a critical task such as requires $24 \times 7$ support, the dominating factor is the Activity type. By comparison, the 'Mobile@Branch-Abroad' scenario (sessions on a mobiles at an enterprise branch abroad) shows very high spatial factor, i.e. location is the main decider in this case.

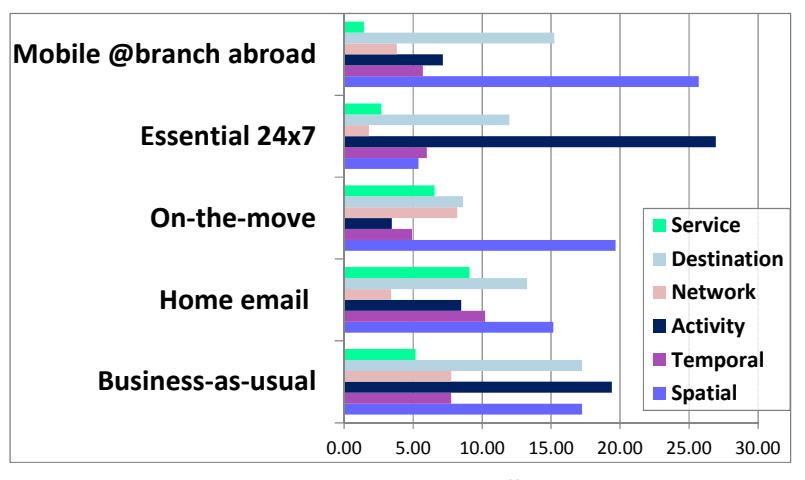

Figure 6: Factors variations in different scenarios

Further analysis is in follow-on papers on Implementing eBC Model and on Modeling Context Attributes.

\section{E. Attributes}

Modeling the relationships between context data items and their relative priorities is studied in [15], which proposes the use of Bayesian model and Directed Acyclic Graph (DAG) to determine probabilities. In this paper we assume that the eBC Function receives probability values for each context item. The more Attributes contribute to a Factor, the more accurate it gets. Greater resilience is also obtained from redundancy, where Attributes can confirm or substitute each other.

Attribute values are generated directly from context data sources. A different set of Attributes belong to each Factor. Several sources can contribute to an Attribute, and have to be consolidated to produce a single figure. Values assigned to an Attribute can be true or false (user is at home or not) or 'fuzzy' (user is near home with 60\% confidence). In [9], Fuzzy Logic is used to select web service composition based on users' preferences. In [11], selection of web service components by QoS parameters is proposed, and Fuzzy Logic is used to simulate human perception of best choice. Similarly here, using fuzzy algorithms can turn imprecise context attribute data into clear-cut value. The eBC Model accepts numeric Attribute values (1-10) as levels of confidence, e.g. the 'at work' Attribute is true if the user is proven to be in the usual place of work $(=10)$, but a fuzzy location reading of 'near work' may be indicated by probability of $=7$ (near) or 9 (extremely near).

$$
\mathrm{Aj}=\max \{\mathrm{PA} 1, \mathrm{PA} 2, \mathrm{PA} 3 \ldots \mathrm{Pam}\}
$$

\section{F. Applying priorities and weighs}

Configuring the priorities for Attributes and weighting ratios for Factors is up to the enterprise staff. Factors and Attributes are prioritized within each Task. Factors values are weighted by ratios (WFi) that represent the Factor significance within the task, totaling 100 across the Task Template. Attributes weights (WAj) represent the enterprise preference and desirability within the Task. For example, for home 
working, service type attribute of email is preferred over video calls and has higher weight WAj in the Home Task.

$$
\begin{aligned}
& 100=\mathrm{WF} 1+\mathrm{WF} 2 \ldots+\mathrm{WFi} \\
& 100=\mathrm{WA} 1+\mathrm{WA} 2 \ldots+\mathrm{WAj}
\end{aligned}
$$

A Factor is a product of a prioritized Attribute probability:

$\mathrm{Fi}=\mathrm{Aj} * \mathrm{WAj} \quad$ where $\mathrm{Aj}$ is the prevailing Attribute

\section{G. The eBC Score}

The sum of weighted Factors determines the score of each Task Template. The prevailing Task has the highest score within the set of allowable templates (TTn).

$$
\mathrm{TTk}=\mathrm{F} 1 * \mathrm{WF} 1+\mathrm{F} 2 * \mathrm{WF} 2 \ldots \mathrm{Fi} * \mathrm{WFi}
$$

The highest TT score becomes the eBC final score for the Role $(\mathrm{eBCn})$ when it is modified by the employee's Grade:

$\mathrm{eBCn}=\max \mathrm{TTn} * \mathrm{Gn}$

This result is compared with a pre-set Threshold (TH) to determine if $\mathrm{eBC}$ status is to be granted:

If $\mathrm{eBCn}>\mathrm{TH} \rightarrow \mathrm{eBC}$ status.

\section{H. PCC Rules}

Since the QoS/Policy profile is determined by the eBC results, the transformation of business objectives into network policies is entirely dependent on the enterprise configuring the eBC Model. The policy parameter levels are pre-configured for each Task Template, according to the task characteristics. In particular, they affect scalar variables such as the QCI (Quality

\begin{tabular}{|c|c|c|c|c|c|c|}
\hline Scalar & Routine & Home & Travel & Essential & Abroad & \\
\hline QCI (Quality Classification ID) & 4 & 3 & 4 & 7 & 5 & \\
\hline Charging key & 4 & 2 & 5 & 8 & 6 & 10: Unlimited \\
\hline MBR (Max Bit Rate) UL & 4 & 5 & 3 & 7 & 4 & $\begin{array}{l}\text { 9: Extra High } \\
\text { 8: Very High }\end{array}$ \\
\hline MBR (Max Bit Rate) DL & 4 & 5 & 5 & 7 & 7 & $\begin{array}{l}\text { 7: High } \\
\text { 6: Medium-High }\end{array}$ \\
\hline GBR (Guaranteed Bit Rate) UL & 5 & 2 & 4 & 8 & 3 & 5: Medium \\
\hline GBR (Guaranteed Bit Rate) DL & 5 & 3 & 4 & 8 & 7 & 3: Low \\
\hline APR (Allocation /Retention Priority) & 3 & 2 & 3 & 7 & 4 & $\begin{array}{l}\text { 2:Very-Low } \\
\text { 1: Extra low }\end{array}$ \\
\hline Revalidation time limit & 7 & 5 & 6 & 7 & 5 & \\
\hline
\end{tabular}
Class ID) or authorized maximum bit rate. Figure 7 provides examples of PCC parameters by Task Templates, where the

Figure 7: PCC Rule levels for Task Templates

values indicate relative levels rather than actual parameters.

The pre-set PCC Rule is interpreted within the carrier's SLA range for each scalar variable, taking into account session requirements, e.g. the service type (high for video, low for text). Fine-tuning is achieved by applying the level of the eBC score to scalar values such as charging, priority, volume capping and time limits.

\section{CONCLUSIONS}

In this paper a practical enterprise-own Business Context function is described, with its platform, data sources and computation. The eBC Function grants Business Context status dynamically, while determining what $\mathrm{PCC}$ rules and charging levels should be assigned. The eBC processing provides several options for conveying policy to carriers. The logic flow is designed to minimize real-time $\mathrm{eBC}$ processing by evaluating static data first and process only relevant templates. Dynamic context evaluation is performed in the eBC Model, as illustrated by results from the proof-of-concept. It uses a hierarchical component structure with Roles, Tasks, Factors and Attributes, linking business objectives with network policy rules. We introduce the idea of Factors to aggregate Attributes and characterize Task Templates. This enables the discovery of a prevailing task and its associated PCC rules.

This eBC Function is conceived for funding decision, but it can apply to other context-dependent processes, where the enterprise can make its own decisions and avoid divulging information externally. The strength of the eBC Function is in interpreting common business scenarios and applying business rules that can promote or discourage behavior. As automating funding decision by dynamic context can save considerable costs, this eBC platform should be commercially viable.

\section{REFERENCES}

[1] A K Malik, S Dustdar "A Hybrid Sharing Control Model for Context Sharing and Privacy in Collaborative Systems" IEEE 2011 ICAINA

[2] 3GPP TS 23.203 Policy and charging control architecture Release11

[3] L H Ngoh, T Y Chai, J C M Teo, M T Wong, Z Lying, S Xu, T K Lee "Policy-based Orchestration of Web/IMS Services" IEEE 2010

[4] I Miloucheva1, D Wagner, C Niephaus, D Hetzer "User-centric identity enabled QoS policy management for Next Generation Internet", I.RE.CO.S.

[5] G Ma, K Wu, T Zhang, W Li “A Flexible Policy-Based Access Control Model for Workflow Management Systems" IEEE 2011

[6] J Strassner, S v-d-Meer, D O'Sullivan, S Dobson "The Use of ContextAware Policies and Ontologies to Facilitate Business-Aware Network Management" Springer, LLC 2009

[7] N Blum, S Lampe, $\mathrm{T}$ Magedanz "Enabling Context-Sensitive Communication Experiences”, IEEE2010

[8] S C Chou, J Y Jhu "Access Control Policy Embedded Composition Algorithm for Web Services" NSC-99-2221-E-259-008

[9] Z Wu, M Yuan "User-Preference-Based Service Selection Using Fuzzy Logic" IEEE CNSM 2010 proceedings

[10] Oasis XACML v3.0 Administration and Delegation Profile Version 1.0

[11] M Sathya, M Swarnamugi, P Dhavachelvan, G Sureshkumar "Evaluation of QoS Based Web-Service Selection Techniques for Service Composition", IJSE 2010, Volume 1 Issue 5

[12] A C O’Connor, R J Loomis "2010 Economic Analysis of Role-Based Access Control" RTI International report for NIST

[13] D Ferraiolo, D Kuhn "Role-Based Access Controls" 15th National Computer Security Conference (1992) Baltimore

[14] R K Thomas, R S Sandhu "Task-based Authorization Controls (TBAC): A Family of Models for Active and Enterprise-oriented Authorization Management" International Conference on Database Securty XI (1998)

[15] K E Ko, K B Sim "Development of Context Aware System based on Bayesian Network driven Context Reasoning Method and Ontology Context Modeling” IEEE ICCAS 2008. 